\title{
Front Matter: Volume 7740
}

, "Front Matter: Volume 7740," Proc. SPIE 7740, Software and Cyberinfrastructure for Astronomy, 774001 (3 August 2010); doi: 10.1117/12.871473

SPIE Event: SPIE Astronomical Telescopes + Instrumentation, 2010, San Diego, SPIE. California, United States 


\title{
PROCEEDINGS OF SPIE
}

\section{Software and Cyberinfrastructure for Astronomy}

\author{
Nicole M. Radziwill \\ Alan Bridger \\ Editors
}

27-30 June 2010

San Diego, California, United States

Sponsored by

SPIE

Cooperating Organizations

American Astronomical Society (United States) - Association of Universities for Research in Astronomy, Inc. (United States) - Astronomical Society of Japan (Japan) • Atacama Large Millimeter /submillimeter Array • Ball Aerospace \& Technologies Corporation (United States) Canadian Astronomical Society (CASCA) (Canada) • Commissariat à l'Energie Atomique (France) • European Astronomical Society (Switzerland) • European Organisation for Astronomical Research in the Southern Hemisphere (Germany) - Japan Aerospace Exploration Agency (Japan) • Jet Propulsion Laboratory (United States) • NASA Goddard Space Flight Center (United States) • National Astronomical Observatory Japan (Japan) National Radio Astronomy Observatory - SOFIA-Stratospheric Observatory for Infrared Astronomy (United States) - Thirty Meter Telescope Project (United States) - W. M. Keck Observatory (United States)

Published by

SPIE

Volume $\mathbf{7 7 4 0}$

Part One of Two Parts

Proceedings of SPIE, 0277-786X, v. 7740 
The papers included in this volume were part of the technical conference cited on the cover and title page. Papers were selected and subject to review by the editors and conference program committee. Some conference presentations may not be available for publication. The papers published in these proceedings reflect the work and thoughts of the authors and are published herein as submitted. The publisher is not responsible for the validity of the information or for any outcomes resulting from reliance thereon.

Please use the following format to cite material from this book:

Author(s), "Title of Paper," in Software and Cyberinfrastructure for Astronomy, edited by Nicole M. Radziwill, Alan Bridger, Proceedings of SPIE Vol. 7740 (SPIE, Bellingham, WA, 2010) Article CID Number.

ISSN 0277-786X

ISBN 9780819482303

Published by

SPIE

P.O. Box 10, Bellingham, Washington 98227-0010 USA

Telephone +1 3606763290 (Pacific Time) · Fax +1 3606471445

SPIE.org

Copyright (C) 2010, Society of Photo-Optical Instrumentation Engineers

Copying of material in this book for internal or personal use, or for the internal or personal use of specific clients, beyond the fair use provisions granted by the U.S. Copyright Law is authorized by SPIE subject to payment of copying fees. The Transactional Reporting Service base fee for this volume is $\$ 18.00$ per article (or portion thereof), which should be paid directly to the Copyright Clearance Center (CCC), 222 Rosewood Drive, Danvers, MA 01923. Payment may also be made electronically through CCC Online at copyright.com. Other copying for republication, resale, advertising or promotion, or any form of systematic or multiple reproduction of any material in this book is prohibited except with permission in writing from the publisher. The CCC fee code is 0277-786X/10/\$18.00.

Printed in the United States of America.

Publication of record for individual papers is online in the SPIE Digital Library.

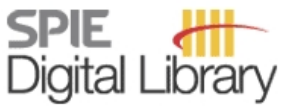

SPIEDigitalLibrary.org

Paper Numbering: Proceedings of SPIE follow an e-First publication model, with papers published first online and then in print and on CD-ROM. Papers are published as they are submitted and meet publication criteria. A unique, consistent, permanent citation identifier (CID) number is assigned to each article at the time of the first publication. Utilization of CIDs allows articles to be fully citable as soon they are published online, and connects the same identifier to all online, print, and electronic versions of the publication. SPIE uses a six-digit CID article numbering system in which:

- The first four digits correspond to the SPIE volume number.

- The last two digits indicate publication order within the volume using a Base 36 numbering system employing both numerals and letters. These two-number sets start with 00, 01, 02, 03, 04, $05,06,07,08,09,0 A, 0 B \ldots 0 Z$, followed by 10-1Z, 20-2Z, etc.

The CID number appears on each page of the manuscript. The complete citation is used on the first page, and an abbreviated version on subsequent pages. Numbers in the index correspond to the last two digits of the six-digit CID number. 


\title{
Contents
}

\section{Part One}

\author{
xix Conference Committee \\ xxi Introduction \\ xxiii Unknowns and unknown unknowns: from dark sky to dark matter and dark energy \\ (Plenary Paper) [7733-501] \\ Y. Suto, The Univ. of Tokyo (Japan)
}

xxxv Optical synoptic telescopes: new science frontiers (Plenary Paper) [7733-502]

J. A. Tyson, Univ. of California, Davis (United States)

\section{CONTROL SYSTEMS I}

774002 Control software and electronics architecture design in the framework of the E-ELT instrumentation [7740-01]

P. Di Marcantonio, I. Coretti, R. Cirami, M. Comari, P. Santin, M. Pucillo, INAF, Osservatorio Astronomico di Trieste (Italy)

774003 Flight control software for the wave-front sensor of SUNRISE 1m balloon telescope [7740-02] A. Bell, Kiepenheuer Institut für Sonnenphysik (Germany); P. Barthol, Max-Planck-Institut für Sonnensystemforschung (Germany); T. Berkefeld, B. Feger, Kiepenheuer Institut für Sonnenphysik (Germany); A. M. Gandorfer, Max-Planck-Institut für Sonnensystemforschung (Germany); F. Heidecke, Kiepenhever Institut für Sonnenphysik (Germany); M. Knoelker, National Ctr. for Atmospheric Research (United States); V. M. Pillet, Instituto de Astrofísica de Canarias (Spain); W. Schmidt, M. Sigwarth, Kiepenhever Institut für Sonnenphysik (Germany); S. K. Solanki, Max-Planck-Institut für Sonnensystemforschung (Germany); D. Soltau, Kiepenhever Institut für Sonnenphysik (Germany); A. M. Title, Lockheed Martin Space Systems Co. (United States)

774004 The LUCIFER control software [7740-03]

M. Jütte, V. Knierim, K. Polsterer, Ruhr-Univ. Bochum (Germany); M. Lehmitz, C. Storz, Max-Planck-Institut für Astronomie (Germany); W. Seifert, Landessternwarte Heidelberg (Germany); N. Ageorges, Max-Planck-Institut für extraterrestrische Physik (Germany)

774005 The LBT real-time based control software to mitigate and compensate vibrations [7740-73] J. Borelli, J. Trowitzsch, M. Brix, M. Kürster, W. Gässler, T. Bertram, F. Briegel, Max-Planck-Institut für Astronomie (Germany) 
774006 Control software architecture for the SALT Robert Stobie Spectrograph [7740-05] A. Koeslag, J. Brink, P. Menzies, South African Astronomical Observatory (South Africa); K. H. Nordsieck, J. Percival, Univ. of Wisconsin-Madison (United States); T. B. Williams, Rutgers, The State Univ. of New Jersey (United States)

774007 Software systems for operation, control, and monitoring of the EBEX instrument [7740-06] M. Milligan, Univ. of Minnesota (United States); P. Ade, Cardiff Univ. (United Kingdom); F. Aubin, McGill Univ. (Canada); C. Baccigalupi, Scuola Internazionale Superiore di Studi Avanzati (Italy); C. Bao, Univ. of Minnesota (United States); J. Borrill, C. Cantalupo, Lawrence Berkeley National Lab. (United States); D. Chapman, J. Didier, Columbia Univ. (United States); M. Dobbs, McGill Univ. (Canada); W. Grainger, Cardiff Univ. (United Kingdom); S. Hanany, Univ. of Minnesota (United States); S. Hillbrand, Columbia Univ. (United States); J. Hubmayr, National Institute of Standards and Technology (United States); P. Hyland, McGill Univ. (Canada); A. Jaffe, Imperial College London (United Kingdom); B. Johnson, Univ. of California, Berkeley (United States); T. Kisner, Lawrence Berkeley National Lab. (United States); J. Klein, Univ. of Minnesota (United States); A. Korotkov, Brown Univ. (United States); S. Leach, Scuola Internazionale Superiore di Studi Avanzati (Italy); A. Lee, Univ. of California, Berkeley (United States); L. Levinson, Weizmann Institute of Science (Israel); M. Limon, Columbia Univ. (United States); K. MacDermid, McGill Univ. (Canada); T. Matsumura, California Institute of Technology (United States); A. Miller, Columbia Univ. (United States); E. Pascale, Cardiff Univ. (United Kingdom); D. Polsgrove, Univ. of Minnesota (United States); N. Ponthieu, Univ. Paris-Sud (France); K. Raach, Univ. of Minnesota (United States); B. Reichborn-Kjennerud, Columbia Univ. (United States); I. Sagiv, Univ. of Minnesota (United States); H. Tran, Univ. California, Berkeley (United States); G. S. Tucker, Y. Vinokurov, Brown Univ. (United States); A. Yadav, M. Zaldarriaga, Institute for Advanced Study (United States); K. Zilic, Univ. of Minnesota (United States)

774008 Faking it for pleasure and profit: the use of hardware simulation at AAO [7740-07] K. Shortridge, M. Vuong, Anglo-Australian Observatory (Australia)

774009 The TJO-OAdM robotic observatory: OpenROCS and dome control [7740-08] J. Colomé, X. Francisco, I. Ribas, K. Casteels, J. Martín, Institut de Ciències de l'Espai (Spain)

\section{REAL-TIME/EVENTS}

7740 OA Heterogeneous real-time computing in radio astronomy (Invited Paper) [7740-09] J. M. Ford, P. Demorest, S. Ransom, U.S. National Radio Astronomy Observatory (United States)

7740 OD Transiting planet search in the Kepler pipeline [7740-12] J. M. Jenkins, NASA Ames Research Ctr. (United States); H. Chandrasekaran, NASA Ames Research Ctr. (United States) and Lawrence Livermore National Lab. (United States): S. D. McCauliff, D. A. Caldwell, P. Tenenbaum, J. Li, T. C. Klaus, M. T. Cote, C. Middour, NASA Ames Research Ctr. (United States)

7740 OE Adapting a publish-subscribe middleware to a RPC response pattern [7740-13] D. A. Morrison, J. M. Johnson, W. M. Keck Observatory (United States) 
7740 OF Lessons learned deploying a second generation Observation Control System for Subaru Telescope [7740-16]

E. Jeschke, T. Inagaki, Subaru Telescope, National Astronomical Observatory of Japan (United States)

7740 OG Data handling and control for the European Solar Telescope [7740-17]

I. Ermolli, INAF, Osservatorio Astronomico di Roma (Italy); F. Bettonvil, Utrecht Univ. (Netherlands); G. Cauzzi, INAF, Osservatorio Astrofisico di Arcetri (Italy); L. Cavaller, Grantecan, S.A. (Spain); M. Collados, Instituto de Astrofísica de Canarias (Spain); P. Di Marcantonio, INAF, Osservatorio Astronomico di Trieste (Italy); F. Paletou, Lab. d'Astrophysique de Toulouse-Tarbes, CNRS, Univ. de Toulouse (France); P. Romano, INAF, Osservatorio Astrofisico di Catania (Italy); J. Aboudarham, Observatoire de Paris à Meudon (France); R. Cirami, INAF, Osservatorio Astronomico di Trieste (Italy); R. Cosentino, INAF, Osservatorio Astrofisico di Catania (Italy); F. Giorgi, INAF, Osservatorio Astronomico di Roma (Italy); M. Lafon, Lab. d'Astrophysique de Toulouse-Tarbes, CNRS, Univ. de Toulouse (France); D. Laforgue, Instituto de Astrofísica de Canarias (Spain); K. Reardon, INAF, Osservatorio Astrofisico di Arcetri (Italy); G. Sliepen, Utrecht Univ. (Netherlands)

$7740 \mathrm{OH}$ Science data quality assessment for the Large Synoptic Survey Telescope [7740-18] R. A. Shaw, National Optical Astronomy Observatory (United States); D. Levine, California Institute of Technology (United States); T. Axelrod, LSST Corp. (United States); R. R. Laher, V. G. Mannings, California Institute of Technology (United States)

7740 Ol LBT data mining leads to increased open shutter time [7740-19] N. Cushing, C. Biddick, D. Thompson, J. Hill, M. De La Peña, T. Leibold, Large Binocular Telescope Observatory (United States)

$7740 \mathrm{OJ}$ An algorithm for the fitting of planet models to Kepler light curves [7740-20] P. Tenenbaum, S. T. Bryson, NASA Ames Research Ctr. (United States); H. Chandrasekaran, NASA Ames Research Ctr. (United States) and Lawrence Livermore National Lab. (United States); J. Li, E. Quintana, J. D. Twicken, J. M. Jenkins, NASA Ames Research Ctr. (United States)

\section{VO/ARCHIVE}

7740 OK Building archives in the virtual observatory era (Invited Paper) [7740-21]

R. L. Plante, Univ. of Illinois at Urbana-Champaign (United States); G. Greene, R. J. Hanisch, Space Telescope Science Institute (United States); T. A. McGlynn, NASA Goddard Space Flight Ctr. (United States); C. J. Miller, Univ. of Michigan, Ann Arbor (United States); D. Tody, National Radio Astronomy Observatory (United States); R. White, Space Telescope Science Institute (United States)

$7740 \mathrm{OL}$ Shannon sampling and nonlinear dynamics on graphs for representation, regularization and visualization of complex data [7740-22]

M. Pesenson, California Institute of Technology (United States); I. Pesenson, Temple Univ. (United States); B. McCollum, California Institute of Technology (United States); M. Byalsky, The Hebrew Univ. of Jerusalem (Israel) 
7740 OM The Kepler DB: a database management system for arrays, sparse arrays, and binary data [7740-23]

S. McCauliff, M. T. Cote, F. R. Girouard, C. Middour, T. C. Klaus, B. Wohler, NASA Ames

Research Ctr. (United States)

7740 ON The WIYN ODI instrument software configuration and scripting [7740-24]

A. K. Yeatts, J. Ivens, D. Harbeck, WIYN Observatory (United States)

774000 Discovery Channel Telescope software key technologies [7740-25]

P. J. Lotz, Lowell Observatory (United States)

7740 OP Future management needs of a "software-driven" science community [7740-26]

K. K. Nilsson, ST-ECF (Germany); O. Möller-Nilsson, Max-Planck-Institut für Astronomie (Germany)

\section{CYBERINFRASTRUCTURE I}

$7740 \mathrm{OQ} \quad$ An observation execution system for next-generation large telescopes [7740-27]

K. Gillies, Space Telescope Science Institute (United States); S. Walker, Gemini Observatory (Chile)

7740 OR Software architecture of the Magdalena Ridge Observatory Interferometer [7740-28] A. Farris, D. Klinglesmith, J. Seamons, N. Torres, New Mexico Institute of Mining and Technology (United States); D. Buscher, J. Young, Univ. of Cambridge (United Kingdom)

7740 OS Designing a high-availability cluster for the Subaru Telescope second generation observation control system [7740-29]

E. Jeschke, T. Inagaki, Subaru Telescope, National Astronomical Observatory of Japan (Japan)

7740 OT Evolution of the VLT instrument control system toward industry standards [7740-30] M. J. Kiekebusch, G. Chiozzi, J. Knudstrup, D. Popovic, European Southern Observatory (Germany); G. Zins, Lab. d'Astrophysique de l'Observatoire de Grenoble (France)

7740 OU Operating a global network of autonomous observatories [7740-31]

P. Kubánek, Univ. de València (Spain), Instituto de Astrofísica de Andalucía CSIC (Spain), and Institute of Physics (Czech Republic); A. J. Castro-Tirado, Instituto de Astrofísica de Andalucía CSIC (Spain); A. de Ugarte Postigo, INAF, Osservatorio Astronomico di Brera (Italy); R. Cunniffe, M. Prouza, Instituto de Astrofísica de Andalucía CSIC (Spain); J. Štrobl, Astronomical Institute (Czech Republic); H. van Heerden, Boyden Observatory (South Africa); J. Gorosabel, Instituto de Astrofísica de Andalucía CSIC (Spain); R. Hudec, Astronomical Institute (Czech Republic) and Czech Technical Univ. (Czech Republic); P. Yock, Auckland Univ. (New Zealand); W. H. Allen, Vintage Lane Observatory (New Zealand); I. Bond, Massey Univ. (New Zealand); G. Christie, Stardome Observatory (New Zealand); S. Guziy, Instituto de Astrofísica de Andalucía CSIC (Spain); L. Hanlon, Univ. College Dublin (Ireland M. Jelínek, Instituto de Astrofísica de Andalucía CSIC (Spain); S. Meehan, Univ. College Dublin (Ireland); C. Polášek, Astronomical Institute (Czech Republic); V. Reglero, Univ. de València (Spain); P. Vitale, Pierre-Auger Observatory South (Argentina) 
7740 OV Achieving reusability in KMOS instrument software through design patterns [7740-32] M. Wegner, B. Muschielok, Univ. Observatory Munich (Germany)

7740 OW Reusing the VLT control system on the VISTA Telescope [7740-33]

D. L. Terrett, Rutherford Appleton Lab. (United Kingdom); M. Stewart, Sulaire Systems (United Kingdom)

7740 0X Evaluating and evolving common services framework for use at W. M. Keck Observatory [7740-34]

J. Johnson, W. M. Keck Observatory (United States); S. Wampler, National Solar Observatory (United States); K. McCann, W. M. Keck Observatory (United States)

7740 OY Integration of SCUBA-2 within the JCMT Observatory Control System [7740-35] C. A. Walther, Joint Astronomy Ctr. (United States); X. Gao, D. Kelly, UK Astronomy Technology Ctr. (United Kingdom); R. D. Kackley, T. Jenness, Joint Astronomy Ctr. (United States)

$7740 \mathrm{OZ}$ Commensal observing with the Allen Telescope array: software command and control [7740-36]

C. Gutierrez-Kraybill, G. K. Keating, Hat Creek Radio Observatory, Univ. of California, Berkeley (United States); D. MacMahon, P. K. G. Williams, Radio Astronomy Lab., Univ. of California, Berkeley (United States); G. Harp, R. Ackermann, T. Kilsdonk, J. Richards, SETI Institute (United States); W. C. Barott, Embry-Riddle Univ. (United States)

\section{WEB 2.0/USER INTERFACES}

$774010 \quad$ Writing Web 2.0 applications for science archives [7740-37]

W. Roby, California Institute of Technology (United States)

774011 Build great web search applications quickly with Solr and Blacklight [7740-38] R. DuPlain, D. S. Balser, National Radio Astronomy Observatory (United States); N. M. Radziwill, James Madison Univ. (United States) and National Radio Astronomy Observatory (United States)

774012 Graphical user interfaces of the dark energy survey [7740-39]

J. Eiting, A. Elliott, K. Honscheid, The Ohio State Univ. (United States); J. Annis,

E. J. Buckley-Geer, W. Wester, Fermi National Accelerator Lab. (United States); M. Haney, W. Hanlon, I. Karliner, J. Thaler, M. Meyer, Univ. of Illinois (United States); M. Bonati, G. Schumacher, Cerro Tololo Inter-American Observatory (Chile); K. W. Kuehn, S. E. Kuhlmann, Argonne National Lab. (United States); T. Schalk, Univ. of California, Santa Cruz (United States); S. Marshall, A. J. Roodman, SLAC National Accelerator Lab. (United States)

774013 User interface software development for the WIYN One Degree Imager (ODI) [7740-40] J. Ivens, A. Yeatts, D. Harbeck, P. Martin, WIYN Observatory (United States)

774014 The use of Flex as a viable toolkit for astronomy software applications [7740-41] K. Gillies, A. Conti, A. Rogers, Space Telescope Science Institute (United States) 
774015 An open source application framework for astronomical imaging pipelines [7740-42] T. Axelrod, Steward Observatory (United States); J. Kantor, LSST Corp. (United States); R. H. Lupton, Princeton Univ. (United States); F. Pierfederici, Space Telescope Science Institute (United States)

774016 Automated calibration and imaging with the Allen Telescope Array [7740-43] G. K. Keating, Radio Astronomy Lab., Univ. of California, Berkeley (United States); W. C. Barott, Embry-Riddle Aeronautical Univ. (United States); M. Wright, Radio Astronomy Lab., Univ. of California, Berkeley (United States)

774017 Kepler Science Operations Center pipeline framework [7740-44] T. C. Klaus, S. McCauliff, M. T. Cote, F. R. Girouard, B. Wohler, C. Allen, C. Middour, D. A. Caldwell, J. M. Jenkins, NASA Ames Research Ctr. (United States)

774018 The Kepler Science Operations Center pipeline framework extensions [7740-45] T. C. Klaus, M. T. Cote, S. McCauliff, F. R. Girouard, B. Wohler, C. Allen, H. Chandrasekaran, S. T. Bryson, C. Middour, D. A. Caldwell, J. M. Jenkins, NASA Ames Research Ctr. (United States)

774019 Data validation in the Kepler Science Operations Center pipeline [7740-46] H. Wu, J. D. Twicken, P. Tenenbaum, B. D. Clarke, J. Li, E. V. Quintana, C. Allen, NASA Ames Research Ctr. (United States); H. Chandrasekaran, Lawrence Livermore National Lab. (United States); J. M. Jenkins, D. A. Caldwell, B. Wohler, F. Girouard, S. McCauliff, M. T. Cote, T. C. Klaus, NASA Ames Research Ctr. (United States)

\section{KEPLER SESSION}

7740 1A Kepler Science Operations Center architecture (Invited Paper) [7740-47] C. Middour, T. C. Klaus, J. Jenkins, D. Pletcher, M. Cote, NASA Ames Research Ctr. (United States); H. Chandrasekaran, Lawrence Livermore National Lab. (United States); B. Wohler, F. Girouard, J. P. Gunter, K. Uddin, C. Allen, J. Hall, K. Ibrahim, B. Clarke, J. Li, S. McCauliff, E. Quintana, J. Sommers, B. Stroozas, P. Tenenbaum, J. Twicken, H. Wu, D. Caldwell, S. Bryson, P. Bhavsar, M. WU, B. Stamper, T. Trombly, C. Page, E. Santiago, NASA Ames Research Ctr. (United States)

7740 1B Semi-weekly monitoring of the performance and attitude of Kepler using a sparse set of targets [7740-48]

H. Chandrasekaran, NASA Ames Research Ctr. (United States) and Lawrence Livermore National Lab. (United States); J. M. Jenkins, J. Li, F. R. Girouard, J. D. Twicken, D. A. Caldwell, C. Allen, S. T. Bryson, T. C. Klaus, M. T. Cote, NASA Ames Research Ctr. (United States);

B. A. Stroozas, Stroozas FlightOps (United States); J. R. Hall, K. Ibrahim, NASA Ames Research Ctr. (United States)

7740 1C Focal plane geometry characterization of the Kepler Mission [7740-49]

P. Tenenbaum, J. M. Jenkins, NASA Ames Research Ctr. (United States) 
7740 1D Selecting pixels for Kepler downlink [7740-50]

S. T. Bryson, J. M. Jenkins, T. C. Klaus, M. T. Cote, E. V. Quintana, J. R. Hall, K. Ibrahim, NASA Ames Research Ctr. (United States); H. Chandrasekaran, Lawrence Livermore National Lab. (United States); D. A. Caldwell, J. E. Van Cleve, M. R. Haas, NASA Ames Research Ctr. (United States)

7740 IE Kepler Mission's focal plane characterization models implementation [7740-51]

C. Allen, T. Klaus, J. Jenkins, NASA Ames Research Ctr. (United States)

\section{CYBERINFRASTRUCTURE II}

$7740 \mathrm{IF}$ The application of cloud computing to the creation of image mosaics and management of their provenance (Invited Paper) [7740-52]

G. B. Berriman, California Institute of Technology (United States); E. Deelman, P. Groth, G. Juve, The Univ. of Southern California (United States)

7740 IG EVALSO: a high-bandwidth communication infrastructure to efficiently connect the ESO Paranal and the Cerro Armazones Observatories to Europe [7740-53]

G. Filippi, European Organisation for Astronomical Research in the Southern Hemisphere (Germany); S. Jaque, Corp. REUNA (Chile); F. Liello, Consortium GARR (Italy); R. Chini, Bochum Ruhr-Univ. Bochum Astronomisches Institut (Germany); F. Utreras, CLARA (Uruguay); A. Wright, European Organisation for Astronomical Research in the Southern Hemisphere (Germany); R. Lemke, Bochum Ruhr-Univ. Bochum Astronomisches Institut (Germany); F. Heissenhuber, European Organisation for Astronomical Research in the Southern Hemisphere (Germany)

7740 1H File-storage cyberinfrastructure for large-scale projects: years before first-light [7740-54] A. S. Jagatheesan, San Diego Supercomputer Ctr. (United States) and iRODS.org (United States); J. Kantor, LSST (United States); R. Plante, National Ctr. for Supercomputing Applications (United States); J. Becla, SLAC National Accelerator Lab. (United States); D. M. Freemon, National Ctr. for Supercomputing Applications (United States); K.-T. Lim, SLAC National Accelerator Lab. (United States); M. Wan, Institute for Neural Computation (United States) and iRODS.org (United States)

$774011 \quad$ CANFAR: the Canadian Advanced Network for Astronomical Research [7740-55] S. Gaudet, N. Hill, National Research Council Canada (Canada); P. Armstrong, Univ. of Victoria (Canada); N. Ball, J. Burke, B. Chapel, National Research Council Canada (Canada); E. Chapin, Univ. of British Columbia (Canada); A. Damian, P. Dowler, National Research Council Canada (Canada); I. Gable, Univ. of Victoria (Canada); S. Goliath, I. Ghiurea, National Research Council Canada (Canada); S. Fabbro, Univ. of Victoria (Canada); S. Gwyn, D. Jenkins, J. Kavelaars, National Research Council Canada (Canada); B. Major, Univ. of Victoria (Canada); J. Ovellette, National Research Council Canada (Canada); M. Paterson, Univ. of Victoria (Canada); M. Peddle, National Research Council Canada (Canada); D. Penfold-Brown, C. Pritchet, Univ. of Victoria (Canada); D. Schade, National Research Council Canada (Canada); R. Sobie, Univ. of Victoria (Canada); D. Woods, Univ. of British Columbia (Canada); A. Yeung, Univ. of Victoria (Canada); Y. Zhang, National Research Council Canada (Canada) 


\section{CURRENT PROJECT OVERVIEWS}

$77401 \mathrm{~J} \quad$ The Australian SKA Pathfinder (ASKAP) software architecture [7740-56]

J. C. Guzman, B. Humphreys, Australia Telescope National Facility, Commonwealth

Scientific and Industrial Research Organisation (Australia)

7740 1K The DECam data acquisition and control system [7740-57]

K. Honscheid, J. Eiting, A. Elliott, The Ohio State Univ. (United States); J. Annis, Fermi National Accelerator Lab. (United States); M. Bonati, National Optical Astronomy Observatories (United States); E. Buckley-Geer, Fermi National Accelerator Lab. (United States);

F. Castander, Institut d'Estudis Espacials de Catalunya (Spain); L. da Costa, Observatorio Nacional (Brazil); M. Haney, W. Hanlon, I. Karliner, Univ. of Illinois (United States); K. Kuehn, S. Kuhlmann, Argonne National Lab. (United States); S. Marshall, SLAC National Accelerator Lab. (United States); M. Meyer, Univ. of Illinois (United States); E. Neilsen, Fermi National Accelerator Lab. (United States); R. Ogando, Observatorio Nacional (Brazil); A. Roodman, SLAC National Accelerator Lab. (United States); T. Schalk, Univ. of California, Santa Cruz (United States); G. Schumacher, National Optical Astronomy Observatories (United States); M. Selen, Univ. of Illinois (United States); S. Serrano, Institut d'Estudis Espacials de Catalunya (Spain); J. Thaler, Univ. of Illinois (United States); W. Wester, Fermi National Accelerator Lab. (United States)

7740 1L ALMA software management and deployment [7740-58] B. E. Glendenning, National Radio Astronomy Observatory (United States); J. Ibsen, Atacama Large Millimeter/Submillimeter Array (Chile); G. Kosugi, National Astronomical Observatory of Japan (Japan); G. Raffi, European Southern Observatory (Germany)

7740 1M Discovery Channel Telescope software development overview [7740-59] P. J. Lotz, D. Greenspan, R. Godwin, Lowell Observatory (United States); P. Taylor, Observatory Sciences Ltd. (United Kingdom)

7740 IN The Large Synoptic Survey Telescope data management overview [7740-60] J. Kantor, T. Axelrod, LSST (United States)

774010 The Large Synoptic Survey Telescope data challenges [7740-61] J. Kantor, LSST (United States)

\section{Part Two}

\section{POSTER SESSION}

7740 1P Development of an analysis framework for HSC and Belle II [7740-62]

S. Mineo, H. Aihara, The Univ. of Tokyo (Japan); R. Itoh, N. Katayama, IPNS (Japan); N. Yasuda, The Univ. of Tokyo (Japan); H. Furusawa, Y. Okura, S. Miyazaki, National Astronomical Observatory of Japan (Japan); C. Loomis, R. H. Lupton, Princeton Univ. (United States)

7740 IR Experience with a new approach for instrument software at Gemini [7740-64] A. Núñez, S. Walker, Gemini Observatory (Chile); S. Goodsell, Gemini Observatory (United States); J. Dunn, National Research Council Canada (Canada); K. Gillies, Space Telescope Science Institute (United States) 
7740 is New architectures support for ALMA common software: lessons learned [7740-65] C. E. Menay, G. A. Zamora, Univ. Tecnica Federico Santa Maria (Chile); R. J. Tobar, European Southern Observatory (Germany); J. A. Avarias, National Radio Astronomy Observatory (United States); K. R. Dahl-Skog, H. H. von Brand, Univ. Tecnica Federico Santa Maria (Chile); G. Chiozzi, European Southern Observatory (Germany)

7740 IT Photometer performance assessment in Kepler science data processing [7740-66] J. Li, C. Allen, S. T. Bryson, D. A. Caldwell, NASA Ames Research Ctr. (United States); H. Chandrasekaran, NASA Ames Research Ctr. (United States) and Lawrence Livermore National Lab. (United States); B. D. Clarke, J. P. Gunter, J. M. Jenkins, T. C. Klaus, E. V. Quintana, P. Tenenbaum, J. D. Twicken, B. Wohler, H. WU, NASA Ames Research Ctr. (United States)

$77401 \mathrm{U}$ Presearch data conditioning in the Kepler Science Operations Center pipeline [7740-67] J. D. Twicken, H. Chandrasekaran, J. M. Jenkins, J. P. Gunter, F. Girouard, T. C. Klaus, NASA Ames Research Ctr. (United States)

7740 IV Design of modular C++ observatory control system: from observatories to laboratories and back [7740-68]

P. Kubánek, Institute of Physics (Czech Republic), Univ. de València (Spain), and Instituto de Astrofísica de Andalúcia CSIC (Spain); M. Prouza, Institute of Physics (Czech Republic);

R. Cunniffe, Instituto de Astrofísica de Andalúcia CSIC (Spain); M. Wildi, Observatory Vermes (Switzerland); J. Štrobl, Astronomical Institute (Czech Republic); I. Kotov, P. O'Connor, Brookhaven National Lab. (United States); M. Jelínek, Instituto de Astrofísica de Andalúcia CSIC (Spain); A. de Ugarte Postigo, INAF, Osservatorio Astronomico di Brera (Italy)

7740 1X Pixel-level calibration in the Kepler Science Operations Center pipeline [7740-70] E. V. Quintana, J. M. Jenkins, B. D. Clarke, SETI Institute (United States); H. Chandrasekaran, Lawrence Livermore National Lab. (United States); J. D. Twicken, SETI Institute (United States); S. D. McCauliff, Orbital Sciences Corp. (United States); M. T. Cote, NASA Ames Research Ctr. (United States); T. C. Klaus, C. Allen, Orbital Sciences Corp. (United States); D. A. Caldwell, SETI Institute (United States); S. T. Bryson, NASA Ames Research Ctr. (United States)

7740 IY New direction in the development of the observation software framework (BOSS) [7740-71] E. Pozna, European Organisation for Astronomical Research in the Southern Hemisphere (Germany); A. Smette, R. Schmutzer, ESO (Chile); R. Abuter, T. P. Duc, European Organisation for Astronomical Research in the Southern Hemisphere (Germany); P. Santin, Osservatorio Astronomico di Trieste (Italy)

$774012 \quad J C M T$ Telescope Control System upgrades for SCUBA-2 [7740-72]

R. Kackley, Joint Astronomy Ctr. (United States); D. Scott, E. Chapin, The Univ. of British Columbia (Canada); P. Friberg, Joint Astronomy Ctr. (United States)

774020 A framework for propagation of uncertainties in the Kepler data analysis pipeline [7740-74] B. D. Clarke, C. Allen, S. T. Bryson, D. A. Caldwell, NASA Ames Research Ctr. (United States);

H. Chandrasekaran, Lawrence Livermore National Lab. (United States); M. T. Cote,

F. Girouard, J. M. Jenkins, T. C. Klaus, J. Li, C. Middour, S. McCauliff, E. V. Quintana,

P. Tenenbaum, J. D. Twicken, B. Wohler, H. WU, NASA Ames Research Ctr. (United States) 
774022 SPHERE data reduction software: first insights into data reduction software development for next-generation instruments [7740-76]

O. Möller-Nilsson, A. Pavlov, M. Feldt, Max-Planck-Institut für Astronomie (Germany)

774023 Photometric analysis in the Kepler Science Operations Center pipeline [7740-77]

J. D. Twicken, B. D. Clarke, S. T. Bryson, P. Tenenbaum, H. Wu, J. M. Jenkins, F. Girouard,

T. C. Klaus, NASA Ames Research Ctr. (United States)

$774024 \quad$ High performance graphical data trending in a distributed system [7740-78]

C. Maureira, A. Hoffstadt, J. López, Univ. Técnica Federico Santa María (Chile); N. Troncoso, Associated Universities, Inc. (Chile) and Univ. Técnica Federico Santa María (Chile);

R. Tobar, European Southern Observatory (Germany); H. H. von Brand, Univ. Técnica Federico Santa María (Chile)

774025 A simple way to build an ANSI-C like compiler from scratch and embed it on the instrument's software [7740-79]

A. Rodríguez Trinidad, R. Morales Muñoz, M. Abril Martí, L. P. Costillo Iciarra, M. C. Cárdenas Vázquez, O. Rabaza Castillo, A. Ramón Ballesta, M. A. Sánchez Carrasco, S. Becerril Jarque, P. J. Amado González, Instituto de Astrofísica de Andalucía CSIC (Spain)

774027 A methodological proposal for the development of an HPC-based antenna array scheduler [7740-81]

R. Bonvallet, A. Hoffstadt, D. Herrera, D. López, R. Gregorio, M. Almuna, Univ. Técnica Federico Santa María (Chile); R. Hiriart, National Radio Astronomy Observatory (United States); M. Solar, Univ. Técnica Federico Santa María (Chile)

774028 Choosing a control system for CCAT [7740-82]

D. L. Terrett, P. Wallace, Rutherford Appleton Lab. (United Kingdom); A. Bridger, D. Kelly, UK Astronomy Technology Ctr. (United Kingdom)

774029 Progress in cancellable multi-threaded control software [7740-84]

K. Shortridge, T. J. Farrell, Anglo-Australian Observatory (Australia)

7740 2A A solution for remote-upgrading field controllers based on FPGA Cyclone 2C35 [7740-85] D. Zhu, Y. Zhu, Nanjing Univ. of Posts and Telecommunications (China); J. Wang, Nanjing Institute of Astronomical Optics \& Technology (China)

$77402 B \quad$ A virtual reality environment for telescope operation [7740-86] L. A. Martínez, J. L. Villarreal, F. Ángeles, A. Bernal, Univ. Nacional Autónoma de México (Mexico)

$77402 \mathrm{C} \quad$ Middleware design and implementation for LSST [7740-87] D. Mills, NOAO (United States); G. Schumacher, CTIO (United States)

$77402 \mathrm{D}$ The research on direct drives control system in the large aperture telescope [7740-88] X. Li, Nanjing Institute of Astronomical Optics \& Technology (China) and Graduate Univ. of Chinese Academy of Sciences (China); Z. Zhang, D. Wang, Nanjing Institute of Astronomical Optics \& Technology (China) 
$77402 \mathrm{E} \quad$ The PANIC software system [7740-89]

J. M. Ibáñez Mengual, M. Fernández, J. F. Rodríguez Gómez, A. J. García Segura, Instituto de Astrofísica de Andalucía CSIC (Spain); C. Storz, Max-Planck-Institut für Astronomie (Germany)

7740 2F Practical considerations for pointing a binocular telescope [7740-90]

M. D. De La Peña, Large Binocular Telescope Observatory (United States); D. L. Terrett, Rutherford Appleton Lab. (United Kingdom); D. Thompson, C. J. Biddick, Large Binocular Telescope Observatory (United States)

$77402 \mathrm{G} \quad$ A high efficient and fast kNN algorithm based on CUDA [7740-91] T. Pei, Y. Zhang, Y. Zhao, National Astronomical Observatories (China)

$77402 \mathrm{H}$ The Blanco Telescope TCS upgrade [7740-92] G. Schumacher, E. Mondaca, M. Warner, M. Martinez, O. Estay, T. M. C. Abbott, Cerro Tololo Inter-American Observatory (Chile)

$774021 \quad$ A prototype of Hyper Suprime-Cam data analysis system [7740-93] H. Furusawa, National Astronomical Observatory of Japan (Japan); N. Yasuda, The Univ. of Tokyo (Japan); Y. Okura, National Astronomical Observatory of Japan (Japan); F. Nakata, Subaru Telescope, National Astronomical Observatory of Japan (United States); S. Mineo, The Univ. of Tokyo (Japan); T. Takata, National Astronomical Observatory of Japan (Japan); M. Tanaka, R. Itoh, N. Katayama, High Energy Accelerator Research Organization (Japan); Y. Komiyama, S. Miyazaki, National Astronomical Observatory of Japan (Japan); Y. Utsumi, National Astronomical Observatory of Japan (Japan) and Graduate School for Advanced Studies (Japan); H. Aihara, The Univ. of Tokyo (Japan); T. Uchida, High Energy Accelerator Research Organization (Japan); R. H. Lupton, Princeton Univ. (United States)

$77402 \mathrm{~K}$ Instrument-specific features within the observation preparation software for LINC-NIRVANA [7740-95]

A. Pavlov, J. Trowitzsch, Max-Planck-Institut für Astronomie (Germany)

$77402 \mathrm{~L} \quad$ Research of remote control for Chinese Antarctica Telescope based on iridium satellite communication [7740-97]

L. XU, S. Yang, Nanjing Institute of Astronomical Optics \& Technology (China)

$77402 \mathrm{M}$ Comparison of several algorithms for celestial object classification [7740-98]

N. Peng, Y. Zhang, Y. Zhao, National Astronomical Observatories (China)

$77402 \mathrm{~N}$ Design and realization of the IP control core in field controllers for LAMOST spectroscopes [7740-99]

J. Wang, Nanjing Institute of Astronomical Optics \& Technology (China); Z. Han, Nanjing Institute of Astronomical Optics \& Technology (China) and Graduate Univ. of Chinese Academy of Sciences (China); Y. Zeng, S. Dai, Z. Hu, Y. Zhu, L. Wang, Y. Hou, Nanjing Institute of Astronomical Optics \& Technology (China)

774020 Approaches for photometric redshift estimation of quasars from SDSS and UKIDSS [7740-100] D. Wang, Y. Zhang, Y. Zhao, National Astronomical Observatories (China) 
$77402 \mathrm{P} \quad$ Synchronization of motor controller and PC system clocks [7740-101]

F. Kittmann, Max-Planck-Institute (Germany) and Univ. of Cologne (Germany); T. Bertram,

F. Briegel, L. Mohr, J. Berwein, Max-Planck-Institute (Germany)

$77402 R \quad$ The ATST base: command-action-response in action [7740-103]

J. Hubbard, B. Goodrich, S. Wampler, National Solar Observatory (United States)

$774025 \quad$ Automated classification of pointed sources [7740-104]

Y. Zhang, Y. Zhao, National Astronomical Observatories (China); H. Zheng, North China Electric Power Univ. (China)

$77402 \mathrm{~T} \quad$ Support vector machines for quasar selection [7740-105]

N. Peng, Y. Zhang, Y. Zhao, National Astronomical Observatories (China)

$77402 \mathrm{U}$ The design of LBT's telemetry source registration [7740-106]

T. Edgin, N. Cushing, Large Binocular Telescope Observatory, The Univ. of Arizona (United States)

7740 2V Design considerations for LBTI observer interface [7740-107]

V. Vaitheeswaran, P. Hinz, C. O'Connell, J. Kraus, Steward Observatory, The Univ. of Arizona (United States)

$77402 \mathrm{~W} \quad$ A multistrategy control system for field controllers of astronomical instruments [7740-108] D. Zhu, Y. Zhu, Nanjing Univ. of Posts and Telecommunications (China)

$77402 X \quad$ A simple and effective algorithm for quasar candidate selection [7740-109] N. Peng, Y. Zhang, T. Pei, Y. Zhao, National Astronomical Observatories (China)

$77402 Y \quad$ An automated algorithm for determining photometric redshifts of quasars [7740-1 10] D. Wang, Y. Zhang, Y. Zhao, National Astronomical Observatories (China)

$77402 Z$ Separating quasars from stars by support vector machines [7740-111] Y. Zhang, National Astronomical Observatories (China); H. Zheng, North China Electric Power Univ. (China); Y. Zhao, National Astronomical Observatories (China)

774031 Calibration of LAMOST spectral analysis [7740-113]

F. F. Wang, A. L. Luo, Y. H. Zhao, National Astronomical Observatories (China)

774032 Control, acquisition, and quick-look software for infrared spectrometers [7740-1 14]

E. Rossetti, Univ. di Bologna (Italy); E. Oliva, INAF, Osservatorio Astrofisico di Arcetri (Italy);

L. Origlia, INAF, Osservatorio Astronomico di Bologna (Italy)

774033 Robustness of LAMOST networked control system [7740-115]

A.-L. Luo, K.-F. Wu, National Astronomical Observatories (China); J. Dong, Univ. of Science and Technology of China (China)

774034 Research of large telescope control system [7740-116]

X. Shuai, Chizhou College (China); Z. Zhang, National Astronomical Observatories, Nanjing Institute of Astronomical Optics \& Technology (China) 
774035 Position measurement of the direct drive motor of Large Aperture Telescope [7740-117] Y. Li, Nanjing Institute of Astronomical Optics \& Technology (China) and Graduate Univ. of Chinese Academy of Sciences (China); D. Wang, Nanjing Institute of Astronomical Optics \& Technology (China)

774036 A control system for LAMOST CCD cameras [7740-118]

X. Deng, J. Wang, J. Dong, Univ. of Science and Technology of China (China); Y. Luo, National Astronomical Observatories (China); G. Liu, H. Yuan, G. Jin, Univ. of Science and Technology of China (China)

774037 The primary mirror system control software for the VST [7740-119]

P. Schipani, INAF, Osservatorio Astronomico di Capodimonte (Italy); L. Marty, INAF, VSTCeN (Italy); F. Perrotta, INAF, Osservatorio Astronomico di Capodimonte (Italy); D. Magrin, INAF, Osservatorio Astronomico di Padova (Italy); S. D'Orsi, INAF, VSTCeN (Italy)

774038 Telescope information service system of LAMOST [7740-120]

S. W. Sun, A.-L. Luo, National Astronomical Observatories (China)

774039 A code generation framework for the ALMA common software [7740-121]

N. Troncoso, Associated Universities, Inc. (Chile) and Univ. Técnica Federico Santa María (Chile); H. H. von Brand, Univ. Técnica Federico Santa María (Chile); J. Ibsen, European Southern Observatory (Chile); M. Mora, Associated Universities, Inc. (Chile) and Univ. Técnica Federico Santa María (Chile); V. Gonzalez, Associated Universities, Inc. (Chile); G. Chiozzi, B. Jeram, H. Sommer, European Southern Observatory (Germany); G. Zamora, Univ. Técnica Federico Santa María (Chile); A. Tejeda, Univ. Católica del Norte (Chile)

7740 3A The interaction between pointing and active optics on the VISTA telescope [7740-122] D. L. Terrett, Rutherford Appleton Lab. (United Kingdom); W. J. Sutherland, Queen Mary, Univ. of London (United Kingdom)

7740 3B Towards a new Mercator Observatory Control System [7740-123]

W. Pessemier, G. Raskin, S. Prins, Katholieke Univ. Leuven (Belgium); P. Saey, Katholieke Hogeschool Sint-Lieven (Belgium) and Katholieke Univ. Leuven (Belgium); F. Merges, J. P. Padilla, H. Van Winckel, C. Waelkens, Katholieke Univ. Leuven (Belgium)

7740 3C A high-availability distributed hardware control system using Java [7740-124] A. F. Niessner, Jet Propulsion Lab. (United States)

7740 3D Zigbee networking technology and its application in Lamost optical fiber positioning and control system [7740-125]

Y. Jin, C. Zhai, Y. Gu, Z. Zhou, X. Gai, Univ. of Science and Technology of China (China)

$77403 E \quad$ Realizing software longevity over a system's lifetime [7740-126]

K. Lanclos, W. T. S. Deich, R. I. Kibrick, S. L. Allen, J. Gates, Lick Observatory, Univ. of California, Santa Cruz (United States)

7740 3F Instrument control software requirement specification for Extremely Large Telescopes [7740-127]

P. J. Young, The Australian National Univ. (Australia); M. J. Kiekebusch, G. Chiozzi, European Southern Observatory (Germany) 
7740 3G Introducing high performance distributed logging service for ACS [7740-128]

J. A. Avarias, National Radio Astronomy Observatory (United States); J. S. López, C. Maureira, Univ. Técnica Federico Santa María (Chile); H. Sommer, G. Chiozzi, European Southern Observatory (Germany)

$77403 \mathrm{H} \quad$ A new control system hardware architecture for the Hobby-Eberly Telescope prime focus instrument package [7740-129]

C. Ramiller, T. Taylor, T. H. Rafferty, M. E. Cornell, M. Rafal, R. Savage, McDonald

Observatory, The Univ. of Texas at Austin (United States)

$774031 \quad$ Integrating a university team in the ALMA software development process: a successful model for distributed collaborations [7740-130]

M. Mora, Associated Universities, Inc. (Chile) and Univ. Técnica Federico Santa María (Chile); J. Ibsen, European Southern Observatory (Chile); G. Chiozzi, European Southern Observatory (Germany); N. Troncoso, Associated Universities, Inc. (Chile) and Univ. Técnica Federico Santa María (Chile); R. Tobar, European Southern Observatory (Germany); M. Araya, National Institute for Research in Computer Science and Control (France); J. Avarias, National Radio Astronomy Observatory (United States); A. Hoffstadt, Univ. Técnica Federico Santa María (Chile)

7740 3J SPHERE instrumentation software in the construction and integration phases [7740-131] A. Baruffolo, INAF, Osservatorio Astronomico di Padova (Italy); P. Bruno, INAF, Osservatorio Astrofisico di Catania (Italy); D. Fantinel, INAF, Osservatorio Astronomico di Padova (Italy); E. Fedrigo, European Southern Observatory (Germany); L. Gluck, Lab. d'Astrophysique de l'Observatoire de Grenoble (France); M. Kiekebusch, European Southern Observatory (Germany); M. Micallef, Lab. d'Astrophysique de l'Observatoire de Grenoble (France); D. Popovic, European Southern Observatory (Germany); B. Salasnich, INAF, Osservatorio Astronomico di Padova (Italy); P. Steiner, Eidgenössische Technische Hochschule, Institut für Astronomie (Switzerland); G. Zins, Lab. d'Astrophysique de l'Observatoire de Grenoble (France)

7740 3K The TJO-OAdM Robotic Observatory: the scheduler [7740-132]

J. Colomé, K. Casteels, I. Ribas, X. Francisco, Institut de Ciències de I'Espai CSIC (Spain)

7740 3L UCam: universal camera controller and data acquisition system [7740-133]

S. A. McLay, N. N. Bezawada, D. C. Atkinson, UK Astronomy Technology Ctr. (United

Kingdom); D. J. Ives, European Southern Observatory (Germany)

$77403 \mathrm{M}$ A software framework for telemetry and data logging, MMT Observatory, Arizona, USA [7740-134]

J. D. Gibson, T. Trebisky, S. Schaller, D. Porter, MMT Observatory, The Univ. of Arizona (United States)

$77403 \mathrm{~N} \quad$ Software for automated testing and characterization of CCDs for Large Synoptic Survey Telescope (LSST) [7740-135]

M. Prouza, Institute of Physics of the ASCR, v.v.i. (Czech Republic); P. Kubánek, Institute of Physics of the ASCR, v.v.i. (Czech Republic) and Univ. de Valencia (Spain); P. O'Connor, I. Kotov, J. Frank, Brookhaven National Lab. (United States); P. Antilogus, Lab. de Physique Nucléaire et des Hautes Énergies, CNRS-IN2P3, Univs, Paris VI and VII (France) 
774030 Software for automated run-time determination of calibration values and hardware capabilities in torrent detector control systems [7740-136]

N. C. Buchholz, P. N. Daly, R. George, M. Hunten, P. Moore, D. Sawyer, National Optical Astronomy Observatory (United States)

$77403 P \quad$ World coordinate system keywords for FITS files from Lick Observatory [7740-137]

S. L. Allen, J. Gates, R. I. Kibrick, Lick Observatory (United States)

$77403 Q \quad$ Re-using the NOCS as a common instrument interface [7740-138]

P. N. Daly, National Optical Astronomy Observatory (United States)

$77403 R \quad$ Upgrading the Gemini secondary mirror micro-controller [7740-139]

M. J. Rippa, J. Soto, M. Sheehan, Gemini Observatory North (United States); C. J. Carter, Thirty Meter Telescope (United States); G. Perez, Gemini Observatory South (Chile); E. James, R. Wyman, C. Nakayama, C. Yamasaki, Gemini Observatory North (United States)

774035 Effect of noise in image restoration of multi-aperture telescope [7740-140]

Z. Zhou, D. Wang, Y. Wang, Y. Wan, Beijing Univ. of Technology (China)

7740 3T Programmable workflow control with rule check on LAMOST [7740-141]

G. Liu, J. Wang, Univ. of Science and Technology of China (China); A.-L. Luo, National Astronomical Observatories (China); X. Deng, J. Dong, G. Jin, Univ. of Science and Technology of China (China)

Author Index 
Downloaded From: https://www.spiedigitallibrary.org/conference-proceedings-of-spie on 26 Apr 2023

Terms of Use: https://www.spiedigitallibrary.org/terms-of-use 


\title{
Conference Committee
}

\author{
Symposium Chairs
}

Masanori lye, National Astronomical Observatory of Japan (Japan)

Douglas A. Simons, Gemini Observatory (United States)

Symposium Cochairs

Mark M. Casali, European Organisation for Astronomical Research in the Southern Hemisphere (Germany)

Kathryn A. Flanagan, Space Telescope Science Institute (United States)

Conference Chairs

Nicole M. Radziwill, James Madison University (United States) and National Radio Astronomy Observatory (United States)

Alan Bridger, UK Astronomy Technology Centre (United Kingdom)

Program Committee

Gianluca Chiozzi, European Organisation for Astronomical Research in the Southern Hemisphere (Germany)

Kim K. Gillies, Space Telescope Science Institute (United States)

Bret D. Goodrich, National Solar Observatory (United States)

Robert I. Kibrick, Lick Observatory (United States)

Hilton A. Lewis, Keck Observatory (United States) and California Association for Research in Astronomy (United States)

Gianni Raffi, European Organisation for Astronomical Research in the Southern Hemisphere (Germany)

David L. Terrett, Rutherford Appleton Laboratory (United Kingdom)

\section{Session Chairs}

1 Control Systems I

Alan Bridger, UK Astronomy Technology Centre (United Kingdom)

2 Control Systems II

Gianluca Chiozzi, European Organisation for Astronomical Research in the Southern Hemisphere (Germany)

3 Real-Time/Events

David L. Terrett, Rutherford Appleton Laboratory (United Kingdom) 
Data Processing Operations

Kyle Lanclos, Lick Observatory (United States)

5 Vo/Archive

Kim K. Gillies, Space Telescope Science Institute (United States)

6 Cyberinfrastructure I

Gianluca Chiozzi, European Organisation for Astronomical Research in the Southern Hemisphere (Germany)

7 Common Services/Reuse

Hilton A. Lewis, Keck Observatory (United States) and California Association for Research in Astronomy (United States)

$8 \quad$ Web 2.0/User Interfaces

Bret D. Goodrich, National Solar Observatory (United States)

10 Pipelines/Kepler

Steve L. Allen, Lick Observatory (United States)

11 Kepler Session

David L. Terrett, Rutherford Appleton Laboratory (United Kingdom)

12 Cyberinfrastructure II

Hilton A. Lewis, Keck Observatory (United States) and California Association for Research in Astronomy (United States)

13 Current Project Overviews

Bret D. Goodrich, National Solar Observatory (United States) 


\section{Introduction}

Thanks to everyone who joined us for the 2010 Software and Cyberinfrastructure for Astronomy conference in San Diego, California. This was an exciting conference that brought together key players in software development for both ground and space based instruments. Although common services and software reuse continues to be a theme, we heard more this year about executing observations using Web technologies, developing and managing pipelines for data processing, and leveraging complex cyberinfrastructure and cloud computing. There was a special section on Kepler, covering the extensive work that has been done for that instrument recently, and updates on the newer projects including ASKAP, DECam, ALMA and LSST.

One highlight of the 2010 conference was the Lightning Talks session held on Tuesday, June 29. A total of 19 speakers presented improvisational presentations or led interactive group discussions in their allotted five minutes. A breadth of topics were covered, including: using Twitter for telescope operations, events and commands, good user interface design, the SPOT proposal tool, using Google Calendar, version control, 3D modeling, and the ideal qualifications for an observatory software specialist. One presenter even shared some software humor that the audience greatly appreciated.

In addition to the many contributors, without whom this meeting would not have been possible, we would also like to thank the program committee for their continued dedication and support. We look forward to continued progress and anticipate that our community will report on even more exciting innovations at our next gathering in Amsterdam in 2012. See you there!

Nicole M. Radziwill Alan Bridger 
Downloaded From: https://www.spiedigitallibrary.org/conference-proceedings-of-spie on 26 Apr 2023

Terms of Use: https://www.spiedigitallibrary.org/terms-of-use 